\title{
Results from the LUX dark matter experiment
}

\author{
Markus Horn ${ }^{\mathrm{a}, *}$,
}

on behalf of the LUX Collaboration:

D.S. Akerib ${ }^{\mathrm{b}}$, H.M. Araújo ${ }^{\mathrm{c}}$, X. Bai ${ }^{\mathrm{d}}$, A.J. Bailey $^{\mathrm{c}}$, J. Balajthy ${ }^{\mathrm{e}}$, E. Bernard ${ }^{\mathrm{a}}$, A. Bernstein ${ }^{\mathrm{f}}$, A. Bradley ${ }^{\mathrm{b}}$, D. Byram ${ }^{\mathrm{g}}$, S.B. Cahn ${ }^{\mathrm{a}}$,

M.C. Carmona-Benitez ${ }^{\text {h, C. Chan }}{ }^{\mathrm{i}}$, J.J. Chapman ${ }^{\mathrm{i}}$, A.A. Chiller ${ }^{\mathrm{g}}$, C. Chillerg,

A. Currie ${ }^{\mathrm{c}}$, L. de Viveiros ${ }^{\mathrm{j}}$, A. Dobi ${ }^{\mathrm{e}}$, J. Dobson $^{\mathrm{k}}$, E. Druszkiewicz ${ }^{\mathrm{l}}$,

B. Edwards ${ }^{\text {a }}$, C.H. Faham ${ }^{\mathrm{m}}$, S. Fiorucci ${ }^{\mathrm{i}}$, C. Flores ${ }^{\mathrm{n}}$, R.J. Gaitskell ${ }^{\mathrm{i}}$, V.M. Gehman ${ }^{\mathrm{m}}$, C. Ghag ${ }^{\mathrm{o}}$, K.R. Gibson ${ }^{\mathrm{b}}$, M.G.D. Gilchriese ${ }^{\mathrm{m}}$, C. Hall ${ }^{\mathrm{e}}$, M. Hanhardt ${ }^{\mathrm{p}}$, S. Haselschwardt ${ }^{\mathrm{h}}$, S.A. Hertel ${ }^{\mathrm{a}}$, D.Q. Huang ${ }^{\mathrm{i}}$, M. Ihm ${ }^{\mathrm{q}}$,

R.G. Jacobsen ${ }^{\mathrm{q}}$, K. Kazkaz ${ }^{\mathrm{f}}$, R. Knoche ${ }^{\mathrm{e}}$, N.A. Larsen ${ }^{\mathrm{a}}$, C. Lee ${ }^{\mathrm{b}}$, B. Lenardo ${ }^{\mathrm{n}}$, K.T. Lesko ${ }^{\mathrm{m}}$, A. Lindote ${ }^{\mathrm{j}}$, M.I. Lopes ${ }^{\mathrm{j}}$, D.C. Malling ${ }^{\mathrm{i}}$, R. Mannino ${ }^{\mathrm{r}}$, D.N. McKinsey a , D.-M. Mei ${ }^{\mathrm{g}}$, J. Mockn ${ }^{\mathrm{n}}$, M. Moongweluwan ${ }^{\mathrm{l}}$, J. Morad ${ }^{\mathrm{n}}$, A.St.J. Murphy ${ }^{\mathrm{k}}$, C. Nehrkorn ${ }^{\mathrm{h}}$, H. Nelson ${ }^{\mathrm{h}}$, F. Neves ${ }^{\mathrm{j}}$, R.A. Ott ${ }^{\mathrm{n}}$, M. Pangilinan ${ }^{\text {i, P.D. Parker }}{ }^{\mathrm{a}}$, E.K. Pease ${ }^{\mathrm{a}}$, K. Pech ${ }^{\mathrm{b}}$, P. Phelps ${ }^{\mathrm{b}}$, L. Reichhart ${ }^{\circ}$, T. Shutt ${ }^{b}$, C. Silva ${ }^{j}$, V.N. Solovov ${ }^{j}$, P. Sorensen ${ }^{f}$,

K. O’Sullivan ${ }^{\mathrm{a}}$, T.J. Sumner ${ }^{\mathrm{c}}$, M. Szydagis ${ }^{\mathrm{n}}$, D. Taylor ${ }^{\mathrm{p}}$, B. Tennyson ${ }^{\mathrm{a}}$, D.R. Tiedt ${ }^{\mathrm{d}}$, M. Tripathi ${ }^{\mathrm{n}}$, S. Uvarov ${ }^{\mathrm{n}}$, J.R. Verbus ${ }^{\mathrm{i}}$, N. Walsh ${ }^{\mathrm{n}}$, R. Webb $^{\mathrm{r}}$,

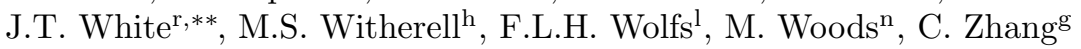

\footnotetext{
${ }^{a}$ Yale University, Dept. of Physics, 217 Prospect St., New Haven CT 06511, USA

${ }^{b}$ Case Western Reserve University, Dept. of Physics, 10900 Euclid Ave, Cleveland OH 44106, USA

${ }^{c}$ Imperial College London, High Energy Physics, Blackett Laboratory, London SW7 2BZ, $U K$

${ }^{d}$ South Dakota School of Mines and Technology, 501 East St Joseph St., Rapid City SD 57701, USA

${ }^{e}$ University of Maryland, Dept. of Physics, College Park MD 20742, USA

${ }^{f}$ Lawrence Livermore National Laboratory, 7000 East Ave., Livermore CA 94551, USA

${ }^{g}$ University of South Dakota, Dept. of Physics, 414E Clark St., Vermillion SD 57069, USA

${ }^{h}$ University of California Santa Barbara, Dept. of Physics, Santa Barbara, CA, USA

${ }^{i}$ Brown University, Dept. of Physics, 182 Hope St., Providence RI 02912, USA

${ }^{j}$ LIP-Coimbra, Department of Physics, University of Coimbra, Rua Larga, 3004-516 Coimbra, Portugal

${ }^{k}$ SUPA, School of Physics and Astronomy, University of Edinburgh, Edinburgh, EH9 3JZ, $U K$

${ }^{l}$ University of Rochester, Dept. of Physics and Astronomy, Rochester NY 14627, USA

${ }^{m}$ Lawrence Berkeley National Laboratory, 1 Cyclotron Rd., Berkeley, CA 94720, USA

${ }^{n}$ University of California Davis, Dept. of Physics, One Shields Ave., Davis CA 95616, USA

${ }^{\circ}$ Department of Physics and Astronomy, University College London, Gower Street, London WC1E 6BT, UK

${ }^{p}$ South Dakota Science and Technology Authority, Sanford Underground Research Facility, Lead, SD 57754, USA

${ }^{q}$ University of California Berkeley, Department of Physics, Berkeley, CA 94720, USA

${ }^{r}$ Texas A 83 M University, Dept. of Physics, College Station TX 77843, USA
}

${ }^{*}$ Corresponding author: markus.horn@yale.edu

** Deceased

Preprint submitted to Elsevier

October 20, 2014

(C) 2014. This manuscript version is made available under the Elsevier user license http://www.elsevier.com/open-access/userlicense/1.0/ 


\begin{abstract}
The LUX (Large Underground Xenon) experiment aims at the direct detection of dark matter particles via their collisions with xenon nuclei. The $370 \mathrm{~kg}$ two-phase liquid xenon time projection chamber measures simultaneously the scintillation and ionization from interactions in the target. The ratio of these two signals provides very good discrimination between potential nuclear recoil and electronic recoil signals to search for WIMP-nucleon scattering. The LUX detector operates at the Sanford Underground Research Facility (Lead, South Dakota, USA) since February 2013. First results were presented in late 2013 setting the world's most stringent limits on WIMP-nucleon scattering cross sections over a wide range of WIMP masses. A 300 day run beginning in 2014 will further improve the sensitivity and new calibration techniques will reduce systematics for the WIMP signal search.

Keywords: dark matter, WIMP, liquid xenon, time projection chamber
\end{abstract}

\title{
1. Introduction
}

The existence of non-baryonic cold dark matter is supported by presently available data from a wide range of cosmological observations. Among those are galactic rotation curves, the precise measurements of the cosmic microwave background, the abundance of light elements, the study of supernovae and the mapping of large scale structures [1]. The identity of dark matter remains unknown and is a question of central importance in both astrophysics and particle physics. A generic weakly interacting massive particle (WIMP) is one of the leading candidates to account for dark matter in our universe. Most direct detection experiments aim to detect WIMPs via low energy nuclear recoils caused by elastic scattering in dedicated low background detectors. The experimental challenge for these experiments is to utilize a large target mass (e.g. LUX has a fiducial volume of $\sim 118 \mathrm{~kg}$ ), to achieve a low energy threshold (typically well 


\footnotetext{
${ }^{1}$ The subscript 'nr' emphasizes true nuclear recoil energies, in comparison to reconstructed energies from electron equivalent recoils expressed in $\mathrm{keV}_{\mathrm{ee}}$.

${ }^{2}$ For more information, please refer to www.luxdarkmatter.org
} 
ture by electronegative impurities, of $87 \pm 9$ and $134 \pm 15 \mathrm{~cm}$ has been achieved. The electron extraction field is $6 \mathrm{kV} / \mathrm{cm}$ (in gas) and $3.1 \mathrm{kV} / \mathrm{cm}$ (in liquid) respectively, yielding to an electron extraction efficiency of $0.65 \pm 0.01$.

The time between the two events (S1 and S2) provide the depth of an event, while the S2 hit pattern of light on the PMT arrays provide the $\mathrm{x}-\mathrm{y}$ position. The position resolution for S2 signals in LUX is determined to 4-6 mm for small S2 signals. Nuclear recoils (NR), in contrast to electron recoils (ER), deposit energy in the material predominantly via heat. Additionally, the ionization-toscintillation ratio of NRs is characteristically reduced with respect to that of ERs. This is the basis for discrimination between NR (from neutrons and the potential WIMP-nucleon interactions) and ER (from background radioactivity) via the ratio of $\mathrm{S} 2$ to $\mathrm{S} 1$ versus $\mathrm{S} 1$. A detailed overview of this phenomenon, a review of measured data and a model of the underlying physics can be found in Ref. [3, 7]. Background rejection with this technique has been shown for LUX to be $\sim 99.6 \%$ in the energy range of interest for WIMP signal search.

The LUX detector is $59 \mathrm{~cm}$ in height and $49 \mathrm{~cm}$ in diameter, lined with polytetrafluoroethylene (PTFE), which has a very high light reflectivity in liquid xenon of greater than 95\%. Together with the PMT's average photon detection efficiency of about 30\%, the reflectivity of the stainless steel grid wires and a finite photon absorption length, this yields to a measured photon detection efficiency for events at the center of LUX of $14 \%$ [8]. This large photon detection efficiency, corresponding to 8.8 phe/keV for a $122 \mathrm{keV}$ gamma at zero-field, allows LUX to be sensitive to WIMP masses above $6 \mathrm{GeV} / \mathrm{c}^{2}$.

\section{First LUX Dark Matter search results}

Starting in April 2013, a total of 85.3 live-days of WIMP search data were taken. The mean background event rate was measured to be $3.6 \pm 0.3 \mathrm{mDRU}$ $\left(\mathrm{mDRU}=10^{-3}\right.$ counts $/ \mathrm{keV} / \mathrm{kg} /$ day $)$ inside the fiducial volume in the energy range of interest (2-30 phe S1 signals), the lowest rate achieved by any xenon TPC so far. Most of those background events are created by residual radioactiv- 

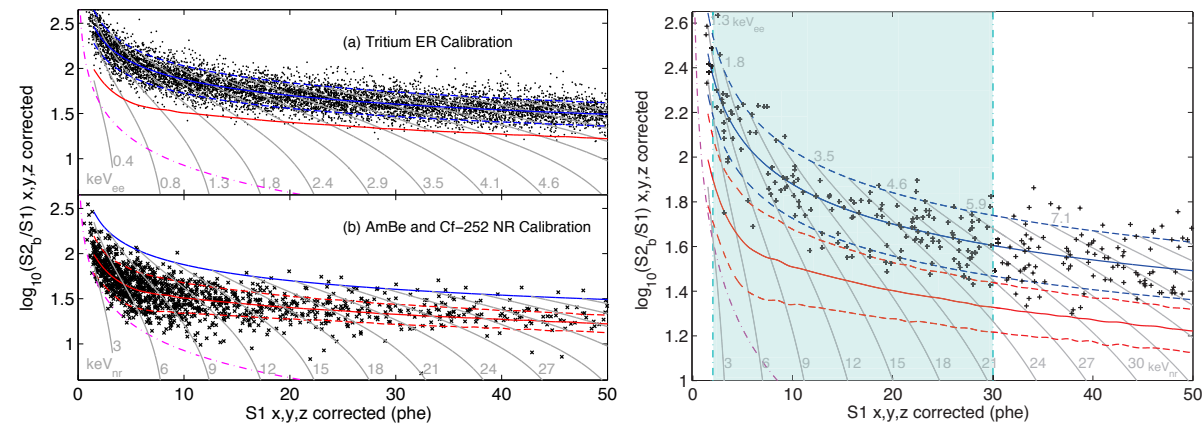

Figure 1: Left: LUX detector response (in $\log _{10}(\mathrm{~S} 2 / \mathrm{S} 1)$ vs $\mathrm{S} 1$ ) in the fiducial volume to calibrations using a tritium internal source (panel a) and $\mathrm{AmBe}$ and ${ }^{252} \mathrm{Cf}$ external sources (panel b), as presented in Ref. 44. The solid lines show the ER mean (blue) and NR mean (red) obtained from simulations together with $\pm 1.28 \sigma$ contours (dashed lines). The 200 phe analysis threshold for S2 signals is shown in both panels as a dashed-dotted line (magenta). Grey contours indicate constant energies in $\mathrm{keV}_{\text {ee }}$ and $\mathrm{keV}_{\mathrm{nr}}$ respectively. Right: The LUX WIMP search data from the 85.3 live-days within the fiducial volume passing all cuts are shown, as presented in Ref. [4. The shaded region indicates the used analysis region from 2-30 phe in S1. The same parameterization of the mean ER and NR bands as on the left are also shown. Please refer to the online-version for color figures. ity of the detector material, primarily the PMTs. Another source of background, intrinsic to our xenon stock, is residual ${ }^{85} \mathrm{Kr}$, a beta emitter at $\mathrm{E}_{\max }=687 \mathrm{keV}$, $\left.\mathrm{T}_{1 / 2}=10.6 \mathrm{yr}\right)$. LUX achieved a measured concentration of $3.5 \pm 1 \mathrm{ppt} \mathrm{Kr}$ before the start of the run by using chromatographic separation of xenon and krypton off-site 4] . During detector operation, an automatic in-line xenon sampling system allows to monitor the Kr level in-situ over time [19]. Further backgrounds are generated from ${ }^{214} \mathrm{~Pb}$ within the ${ }^{222} \mathrm{Rn}$ chain and cosmogenically produced radioisotopes within the xenon itself, namely x-rays from ${ }^{127} \mathrm{Xe}$

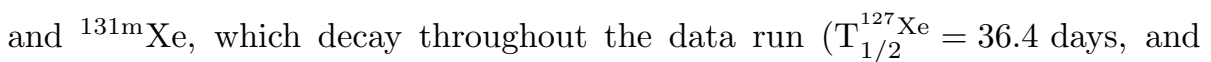
$\mathrm{T}_{1 / 2}^{131 \mathrm{~m}} \mathrm{Xe}=11.9$ days respectively). A detailed study of the radiogenic back- 

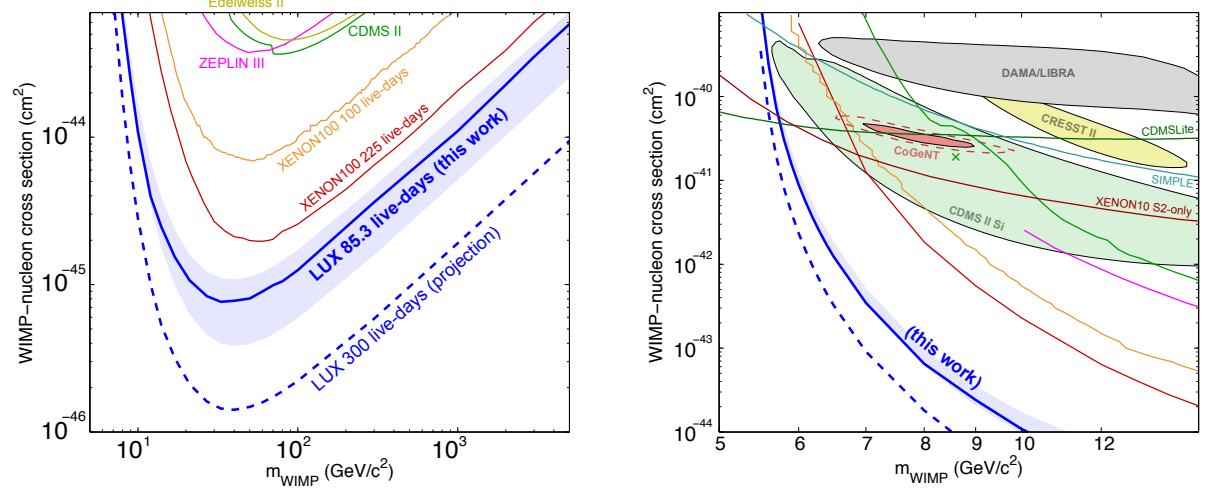

Figure 2: Left: The LUX 90\% C.L. on the spin-independent WIMP-nucleon cross section (solid blue) and a projected limit of the upcoming 300 live-days run (dashed blue). The shaded region indicates $\pm 1 \sigma$ variation from repeated trials, where trials fluctuating below the expected number of background events are forced from zero to 2.3 (blue shaded). Also shown are results from XENON-100 9, 10, ZEPLIN-III [11, CDMS-II [12] and EdelweissII [13. Right: Close-up view at lower WIMP masses together with regions measured by other experiments, e.g. CoGeNT [14] (red), CDMS-II Si [15] (green and 'x'), CRESST-II [16] (yellow) and DAMA/LIBRA [17, 18] (grey). Limits been calculated assuming an artificial cut-off of light yield for nuclear recoils below $3 \mathrm{keV}_{\mathrm{nr}}$, despite evidence of signals down to 0.7 $\mathrm{keV}_{\mathrm{nr}}$. See text for details. Please refer to the online-version for color figures.

grounds in LUX and comparison to simulations can be found in Ref. 20]. The LUX detector was calibrated extensively using internal and external sources. tritiated methane is injected into the xenon circulation system and subsequently removed by the purification system using a hot getter. This allowed a high statistic, homogenous distribution of low energy depositions from $\beta^{-}$events within the liquid xenon $\left(\mathrm{E}_{\max }^{\mathrm{H}_{3}}=18.6 \mathrm{keV}\right)$. The detector response to these ER events, in terms of S1 and S2 signals, are shown in the top left panel of Fig. 1. More frequent calibrations, to monitor the electron drift attenuation length, the light yield and to establish 3D position reconstruction corrections, were performed using ${ }^{83 \mathrm{~m}} \mathrm{Kr}$ with mono-energetic energy depositions at $9.4 \mathrm{keV}$ and $32.1 \mathrm{keV}$. For NR, external AmBe and ${ }^{252} \mathrm{Cf}$ sources were used for calibration. The equiv- 
alent detector response to $\mathrm{NR}$ is shown in the lower left panel of Fig. 1. Also shown in Fig. 1 are the mean and $\pm 1.28 \sigma$ ER and NR band parameterizations derived from the comprehensive NEST simulation model [7].

An unblind analysis with only minimal cuts on the WIMP search data was performed to maintain a high acceptance. Besides detector stability cuts, including xenon pressure, applied voltage and liquid level, only single scatter interactions with one S1 and one S2 in the liquid xenon volume were considered. Energy cuts for the 3D position corrected S1 signal were done by the pulse area (2-30 phe), corresponding to energies of $3-25 \mathrm{keV}_{\mathrm{nr}}$ or $0.9-5.3 \mathrm{keV}_{\text {ee }}$ sing traditional energy estimators as described in Ref. 21] for nuclear and electron recoils respectively. Despite the low NR scintillation light yield assumed (a conservative and unphysical cutoff at $3 \mathrm{keV}_{\mathrm{nr}}$ was assumed), LUX achieved a very good WIMP detection efficiency (roughly $17 \%$ at $3 \mathrm{keV}_{\mathrm{nr}}, 50 \%$ at $4.3 \mathrm{keV}_{\mathrm{nr}}$ and greater than $95 \%$ above $7.5 \mathrm{keV}_{\mathrm{nr}}$ ). The fiducial volume was set to the inner $18 \mathrm{~cm}$ radius and approx. $40 \mathrm{~cm}$ height (electron drift lengths between 38-305 $\mu \mathrm{s}$ ) and calculated to be $118.3 \pm 6.5 \mathrm{~kg}$. Last, an analysis threshold to exclude very small S2 signals was set to 200 phe (corresponding to approx. 8 extracted electrons). For more details on threshold and efficiency studies, as well as the fiducial volume selection, please refer to Ref. [22].

A total of 160 events passed the selection cuts, as shown within the shaded area in the right panel in Fig. 1. The distribution of events is consistent with the expected ER background in both the $\log _{10}(\mathrm{~S} 2 / \mathrm{S} 1)$ and position within the volume. A profile likelihood ratio (PLR) analysis was used to assign a probability as a function of S1, S2 and the r-z position of these events, and compared with the distributions of backgrounds in these variables, as well as simulated distributions of a recoil spectrum for WIMP masse ${ }^{3}$ from 5.5 to $5000 \mathrm{GeV} / \mathrm{c}^{2}$. The resulting 90\% C.L. upper limits of the PLR analysis on the spin-independent WIMP-nucleon interaction cross-sections are shown in Fig. 2, with a close-up

\footnotetext{
${ }^{3}$ A standard isothermal Maxwellian velocity distribution model is used for the dark matter halo, with $\mathrm{v}_{0}=220 \mathrm{~km} / \mathrm{s}, \mathrm{v}_{\text {esc }}=544 \mathrm{~km} / \mathrm{s}, \rho_{0}=0.3 \mathrm{GeV} / \mathrm{cm}^{3}$ and $\mathrm{v}_{\odot}=245 \mathrm{~km} / \mathrm{s}$.
} 
view on low WIMP masses (below $\sim 15 \mathrm{GeV}$ ) on the right panel. These limits show significant improvement to previous presented Dark Matter search experiments (see figure caption for details) and are also in tension with experiments observing potential low-mass WIMP events. The presented limit is lower than past xenon-based experiments, especially for low WIMP masses, despite an assumed cut-off of light yield for nuclear recoils. This is a result of a high S1 light collection efficiency, lower S1 threshold and a comparable S2 threshold. Additionally, the sensitivity for lower mass WIMPs is due to those potential events appearing lower in the $\log _{10}(\mathrm{~S} 2 / \mathrm{S} 1)-\mathrm{S} 1$ plane. The expectation value for the S1 signal is below the threshold and detection would be exclusively due to upwards fluctuations in S1. As a result, the event would appear further from the ER band, leading to high sensitivity even with falling detection efficiency for low energy recoils.

\section{Conclusions and outlook}

The LUX WIMP exclusion limit was derived using a conservative approach on xenon response to NR at low energies. But due to its large exposure (85.3 days with $118 \mathrm{~kg}$ ), as well as a very low threshold and high light collection efficiency, the LUX experiment has achieved the most sensitive spin-independent WIMP exclusion limits to date over a wide range of WIMP masses. The experiment did not observe any potential low-mass WIMP signal as suggested in other experiments, e.g. DAMA [17, 18, CoGeNT [14, CRESST [16] and CDMS Si [15]. LUX will continue taking data, starting 2014, with the goal of 300 live days to further improve the sensitivity by a factor of 5 . It will also benefit, especially towards low-mass WIMP recoil spectra, from new measurements of the xenon response to nuclear recoils using a DD generator. Hereby, mono-energetic neutrons are directed to the detector from outside the water shield. The nuclear recoil energy is determined by reconstructing multiple scatter events within the active region of the detector. Signals for the ionization and scintillation channel are available down to $0.7 \mathrm{keV}_{\mathrm{nr}}$ [23. The inclusion of a low energy recoil 
scintillation yield, directly translates to a lower WIMP-mass sensitivity of the LUX detector, although with limited efficiency. A preliminary analysis results in a change of the slope of the set limit towards lower WIMP-masses and further strengthens the validity of this work using a conservative artificial cut-off at $3 \mathrm{keV}_{\mathrm{nr}}$ and increases the conflict with experiments observing events in this mass range. Designs and plans for a next generation experiment, called LUXZEPLIN (or short LZ), are already in place. The projected improvement in exposure will be up to a factor of $\sim 200$, whilst also lowering the background rate, to achieve a WIMP-nucleon cross section sensitivity of $\sim 2.2 \times 10^{-48} \mathrm{~cm}^{2}$ $\left(\right.$ at $\left.\mathrm{M}_{\mathrm{WIMP}}=50 \mathrm{GeV} / \mathrm{c}^{2}\right)$.

\section{Acknowledgments}

This work was partially supported by the U.S. Department of Energy (DOE) under award numbers DE-FG02- 08ER41549, DE-FG02-91ER40688, DE-FG0295ER40917, DE-FG02-91ER40674, DE-NA0000979, DE-FG02-11ER41738, DESC0006605, DE-AC02-05CH11231, DE-AC52-07NA27344, and DE-FG01-91ER40618; the U.S. National Science Foundation under award numbers PHYS-0750671, PHY-0801536, PHY-1004661, PHY-1102470, PHY-1003660, PHY-1312561, PHY1347449; the Research Corporation grant RA0350; the Center for Ultra-low Background Experiments in the Dakotas (CUBED); and the South Dakota School of Mines and Technology (SDSMT). LIP-Coimbra acknowledges funding from Fundação para a Cîencia e Tecnologia (FCT) through the project-grant CERN/FP/123610/2011. Imperial College and Brown University thank the UK Royal Society for travel funds under the International Exchange Scheme (IE120804). The UK groups acknowledge institutional support from Imperial College London, University College London and Edinburgh University, and from the Science \& Technology Facilities Council for PhD studentship ST/K502042/1 (AB). The University of Edinburgh is a charitable body, registered in Scotland, with registration number SC005336. This research was conducted using computational resources and services at the Center for Computation and Visualization, 
Brown University.

We acknowledge the work of the following engineers who played important roles during the design, construction, commissioning, and operation phases of LUX: S. Dardin from Berkeley, B. Holbrook, R. Gerhard, and J. Thomson from UC Davis, and G. Mok, J. Bauer, and D. Carr from Livermore. We gratefully acknowledge the logistical and technical support and the access to laboratory infrastructure provided to us by the Sanford Underground Research Facility (SURF) and its personnel at Lead, South Dakota. SURF was developed by the South Dakota Science and Technology authority, with an important philanthropic donation from T. Denny Sanford, and is operated by Lawrence Berkeley National Laboratory for the Department of Energy, Office of High Energy Physics.

\section{References}

\section{References}

[1] J. Beringer, et al., Review of Particle Physics, Phys. Rev. D 86 (2012) 010001.

[2] V. Chepel, et al., Liquid noble gas detectors for low energy particle physics, JINST 8 (0) (2013) 4001.

[3] E. Aprile, et al., Liquid xenon detectors for particle physics and astrophysics, Reviews of Modern Physics 82 (2010) 2053.

[4] D. S. Akerib, et al., First Results from the LUX Dark Matter Experiment at the Sanford Underground Research Facility, Phys. Rev. Lett. 112 (9) (2014) 091303.

[5] D. S. Akerib, et al., An ultra-low background PMT for liquid xenon detectors, Nucl. Instr. Meth. 703 (2013) 1-6.

[6] D. S. Akerib, et al., The Large Underground Xenon (LUX) experiment, Nucl. Instr. Meth. 704 (2013) 111-126. 
[7] M. Szydagis, et al., Enhancement of NEST capabilities for simulating lowenergy recoils in liquid xenon, JINST 8 (1).

[8] D. S. Akerib, et al., Technical results from the surface run of the LUX dark matter experiment, Astropart. Phys. 45 (2013) 34-43.

[9] E. Aprile, et al., First Dark Matter Results from the XENON100 Experiment, Phys. Rev. Lett. 105 (2010) 131302.

[10] E. Aprile, et al., Dark Matter Results from 225 Live Days of XENON100 Data, Phys. Rev. Lett. 109 (18) (2012) 181301.

[11] D. Y. Akimov, et al., WIMP-nucleon cross-section results from the second science run of ZEPLIN-III, Phys. Lett. B 709 (1-2) (2012) 14-20.

[12] Z. Ahmed, et al., Dark Matter Search Results from the CDMS II Experiment, Science 327 (5973) (2010) 1619-1621.

[13] E. Armengaud, et al., Final results of the EDELWEISS-II WIMP search using a 4-kg array of cryogenic germanium detectors with interleaved electrodes, Phys. Lett. B 702 (5) (2011) 329-335.

[14] C. E. Aalseth, et al., CoGeNT: A search for low-mass dark matter using p-type point contact germanium detectors, Phys. Rev. D 88 (1) (2013) 012002.

[15] R. Agnese, et al., Silicon Detector Dark Matter Results from the Final Exposure of CDMS II, Phys. Rev. Lett. 111 (25) (2013) 251301.

[16] G. Angloher, et al., Results from $730 \mathrm{~kg}$ days of the CRESST-II Dark Matter search, Eur. Phys. J. C 72 (4).

[17] R. Bernabei, et al., First results from DAMA/LIBRA and the combined results with DAMA/NaI, Eur. Phys. J. C 56 (3) (2008) 333-355.

[18] C. Savage, et al., Compatibility of DAMA/LIBRA dark matter detection with other searches, J. Cosm. Astrop. Phys. 04 (4). 
[19] D. Leonard, et al., A simple high-sensitivity technique for purity analysis of xenon gas, Nucl. Instr. Meth. 621 (1-3) (2010) 678-684.

[20] D. S. Akerib, et al., Radiogenic and Muon-Induced Backgrounds in the LUX Dark Matter Detector, arXivarXiv:1403.1299v1.

[21] C. E. Dahl, The physics of background discrimination in liquid xenon, and first results from Xenon10 in the hunt for WIMP dark matter, Ph.D. thesis, Princeton University (2009).

[22] M. Szydagis, et al., A Detailed Look at the First Results from the Large

घ Underground Xenon (LUX) Dark Matter Experiment, arXivarXiv:1402. 3731 .

[23] J. R. Verbus, Recent Results from the LUX Dark Matter Search, in: Lake Louise Winter Institute 2014, 16-22 February 2014, 2014. 